\title{
Análisis del uso del razonamiento Bayesiano en estudiantes de básica primaria' ${ }^{1}$
}

\section{Analysis of the use of Bayesian reasoning in elementary school students}

\section{Análise do uso do raciocínio Bayesian em alunos do ensino fundamental}

Recibido: mayo de 2013

Aceptado: agosto de 2013
Karen Yissed Torres Mondragón ${ }^{2}$

Ginneth Viviana Parra López ${ }^{3}$

\begin{abstract}
Resumen
Diversas investigaciones sobre la enseñanza de la probabilidad han podido determinar que para las personas es difícil contar hechos en los cuales se privilegie la incertidumbre y a su vez incentive la capacidad para interpretar, comunicar y evaluar situaciones probabilísticas; esto ocurre porque la población está educada bajo la lógica determinista, la cual consiste en que el individuo siempre espere un mismo resultado, y este sea predecible, o bivalente; es decir, que corresponde a responder con un sí o un no. Se presenta un trabajo que centró la mirada en el análisis del razonamiento Bayesiano no como el hecho de utilizar algoritmos, sino como el tomar decisiones en situaciones de incertidumbre para estudiantes de básica primaria.
\end{abstract}

Palabras clave: Razonamiento bayesiano; método multivariado; relato; proyecto de trabajo estadístico; matemáticas escolares; incertidumbre y probabilidad.

\begin{abstract}
Several investigations on the teaching of probability has been determined that it is difficult for people who have made the focus on the uncertainty and in turn encourage the ability to interpret, communicate and evaluate probabilistic situations, this occurs because the population is educated under the deterministic logic, which is that the individual always expect the same result, and this is predictable, or bivalent, ie corresponding to answer yes or no. We present a gaze focused work on the analysis of Bayesian reasoning not like the fact of using algorithms, but as the decision-making under uncertainty for primary school students.
\end{abstract}

Keywords: Bayesian Reasoning; multivariate method; story; project statistical work school mathematics; uncertainty and probability.

\footnotetext{
1 Articulo de Investigación
}

2 Universidad Distrital Francisco José de Caldas. Bogotá, Colombia. Contacto: karentorres24@hotmail.com

3 Universidad Distrital Francisco José de Caldas. Bogotá, Colombia. Contacto: ginneth1226@hotmail.com 


\section{Resumo}

Várias investigações sobre o ensino de probabilidade foi determinado que é difícil para as pessoas que fizeram o foco na incerteza e, por sua vez estimular a capacidade de interpretar, comunicar e avaliar as situações probabilísticas, isso ocorre porque a população é educada sob a lógica determinista, o que é que o indivíduo sempre esperar o mesmo resultado, e é previsível ou bivalentes, isto é, correspondendo a responder sim ou não. Nós apresentamos um olhar trabalho focado na análise de raciocínio Bayesian não como o fato de usar algoritmos, mas como a tomada de decisões em condições de incerteza para os alunos do ensino fundamental.

Palavras chave: Raciocínio Bayesian; método multivariado; história; projeto estatística matemática escolar de trabalho; incerteza e probabilidade.

\section{Presentación del problema}

Existen diversos hechos tanto empíricos como teóricos que permiten reconocer algunas tensiones que se encuentran inmersas en la enseñanza de la probabilidad. En primera medida, los estándares básicos de competencias en matemáticas (2006),en lo referido al pensamiento aleatorio y sistemas de datos enfocados en los grados de básica primaria, evidencian cierto privilegio por la probabilidad clásica. Se espera que otros métodos cómo lo es el Bayesiano sea suficiente en la educación estadística, reuniendo varias ventajas que aunque estén referidas a la investigación, aplican a la enseñanza, como lo afirman algunos autores:

- El método Bayesiano hace uso de toda la información previa disponible, mientras que en inferencia clásica esta información no se tiene en cuenta. Puesto que el investigador especifica la distribución inicial, el enfoque Bayesiano tiene en cuenta la perspectiva del investigador, su conocimiento del problema. (Godino, 2006)

- La inferencia Bayesiana proporciona un método totalmente general, debido a que su aplicación no requiere un tipo particular de distribución y no precisa deducir las distribuciones en el muestreo (Diaz \& Batanero, 2006).
En la aplicación de los métodos clásicos se prioriza el uso de los algoritmos, que como afirma Batanero (2002) "se observa una fuerte tendencia a privilegiar el entrenamiento en formas de calcular, como actividad principal y no la contextualización en un entorno específico dado.... Se espera que a través del uso del relato como recurso didáctico, permita que los estudiantes analicen e interpreten una información a priori, con un lenguaje comprensible y contextualizando la situación, para así poder deducir con certeza la probabilidad de un evento.

La implicación de utilizar los algoritmos en la enseñanza de la probabilidad, hace que los estudiantes permanezcan bajo la lógica determinista (no tienen en la incertidumbre, el azar, lo aleatorio) o en la lógica bivalente (responder con un sí o un no) (Batanero, Ortiz, \& Serrano, 2002). Se espera que los estudiantes trabajen desde el razonamiento estadístico, para que los estudiantes sean capaces de interpretar, discutir, comunicar y evaluar información estadística.

Surgiendo la siguiente pregunta orientadora: ¿Qué elementos del razonamiento estadístico son observables en el proceso de aprendizaje, cuando se utilizan los relatos como recurso didáctico en la enseñanza de la probabilidad Bayesiana, en la educación básica primaria? 


\section{Objetivo general}

Describir y analizar los resultados de la trayectoria que recorren los estudiantes cuando se enfrentan al uso de la probabilidad Bayesiana.

Y los objetivos específicos centrados en diseñar, gestionar, evaluar una actividad que utilice el concepto de probabilidad Bayesiana en la básica primaria, utilizando como recurso didáctico el relato y a analizar los efectos del relato como recurso didáctico.

\section{Referentes teóricos.}

Esta propuesta estará sustentada a partir dos enfoques: el saber y la didáctica. Se presenta el método bayesiano como saber, basándose en la tesis Doctoral: Evaluación de conocimientos y recursos didácticos en la formación de profesores sobre probabilidad condicional (García, Evaluación de conocimientos y recursos didácticos en la formación de profesores sobre probabilidad condicional., 2011, págs. 24,54), para poder determinar que dicho método se define como:

Sean n sucesos disjuntos $B_{1}, B_{2}, \ldots . B_{n}, A \in \Omega$ tales que $P\left(B_{i}\right)>0, P(A)>0$;

$i=1,2, \ldots, n ;$ tales que forman un completo de sucesos. Se verifica que:

$$
P(B i / A)=\frac{P(A / B i) P(B i)}{\sum_{i} P(B i) P(A / B i)}=\frac{P(A / B i) P(B i)}{P(A)}
$$

Cuadras y Cols $\left(1988\right.$, pág. 22), define los sucesos $\left\{B_{1} B_{2}, \ldots B_{n}\right\} \in \Omega$ con $\left(P\left(B_{i}\right)>0\right)$, con una partición en el espacio muestral $E$. A estos sucesos los denominan "causas" $y$ al suceso $A$ "efecto". A es un suceso cualquiera que si ocurre, lo hace conjuntamente con uno de los sucesos de la partición $\left\{B_{1} B_{2}, \ldots B_{n}\right\}$.

Sin embargo dada la importancia del teorema de Bayes en el momento de tomar una decisión, la educación probabilística tal vez ha fijado su enseñanza en grados superiores o universitarios y en taras específicas como la pertinencia de cierto evento o no (Krauss, Bruckmaier, \& Martignon, 2010); obviando su uso tanto en grados menores y en actividades del contexto del estudiante con actividades comunes para él. Como lo dice Krauss, Bruckmaier y Martignon (2010) “...Este concepto conduce a una mejora de las técnicas de razonamiento probabilístico y su representación", siendo el razonamiento bayesiano un gran motor que probablemente se debe generar desde los grados iniciales, donde los alumnos pueden observar la importancia de inferir teniendo en cuenta algunas condiciones.

Por último el docente tendrá en cuenta dicho saber para poder transmitirlo a través de un proyecto de trabajo estadístico definido como lo propone (Rocha, Educación estocástica didáctica de la probabilidad y estadística, 2007):

Son las diferentes acciones de diseño, gestión y evaluación que realiza el profesor para que sus estudiantes se puedan enfrentar a la solución de un problema exitosamente ubicado en un contexto aplicado donde enseñe algún objeto de estudio estocástico. Un proceso de estudio dirigido, formalizado en el entorno del aula de clase y en una institución escolar es lo que puede ser estudiado cuando se indaga en el relación a la REA (Ruta de Estudio y Aprendizaje en el aula), para lo cual el EOS (Enfoque Ontosemiótico en los procesos de instrucción estadística) propone y define criterios de idoneidad didáctica en lo concerniente a las acciones del profesor, denominada Ruta Docente (grupo Crisálida) o trayectoria docente (Godino, 2002).

Este proyecto de aula tiene en cuenta a la teoría de situaciones didácticas, donde se presentará una situación fundamental del razonamiento bayesiano, que permitirá que se dé las diferentes situaciones (acción, formulación. validación e institucionalización), para que los docentes teniendo el saber sabio pueda hacer un tratamiento al mismo y convertirlo en saber a enseñar y que los estudiantes queden con un saber enseñado (Brousseau, 1986).

\section{Metodología}

Este trabajo se dirigió en tres fases con diferente temporalización:

Fase I (Preparación): En el diseño de la actividad se hace la aplicación de la Teoría de situaciones didácticas de (Brousseau, 1986); quien plantea que 
a partir de una situación fundamental, que enmarca el objeto estadístico a trabajar, se desarrolle bajo las situaciones de acción, formulación, validación e institucionalización con el fin de que el estudiante se apropie de la situación y construya el objeto estadístico deseado.

Fase II (Recolección de información): Se desarrolla a partir de la aplicación del proyecto de trabajo estadístico en el aula, ya a partir de este se haga la recolección de la información a 70 alumnos de grado primero y tercero de primaria. Para ello se realiza la transcripción de las hojas de trabajo que especifica el trabajo de los estudiantes.

Fase III (Sistematización y análisis): En cuanto a esta fase, se analizará utilizando el Análisis Multivariado Cluster, es una técnica estadística cuya finalidad es dividir un conjunto de objetos en grupos, donde los perfiles de los objetos en un mismo grupo son similares entre sí (cohesión interna del grupo) y los de los objetos, o con casos atípicos (aislamiento externo del grupo).

\section{Análisis de resultados}

Bajo el análisis multivariado Cluster, y las categorías establecidas de análisis, se generan los siguientes

Figura 1 (Primero)

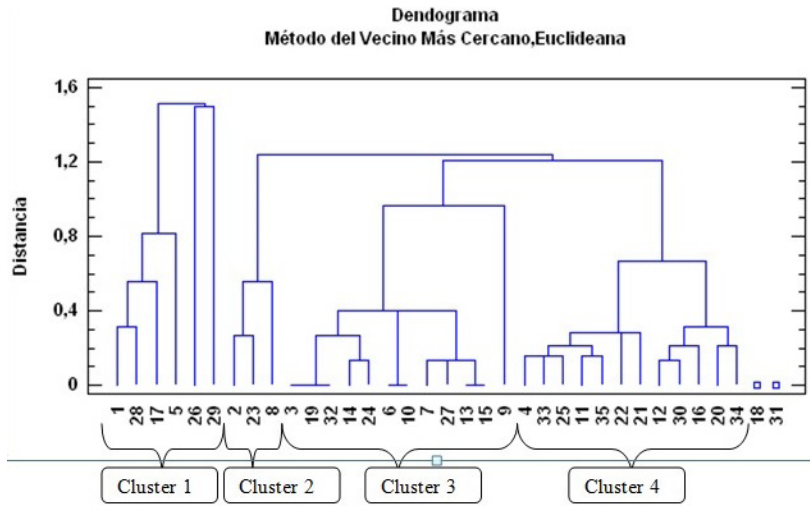

Fuente: Elaboración propia
Figura 2 (Tercero)

Dendograma

Método del Vecino Más Cercano,Euclideana

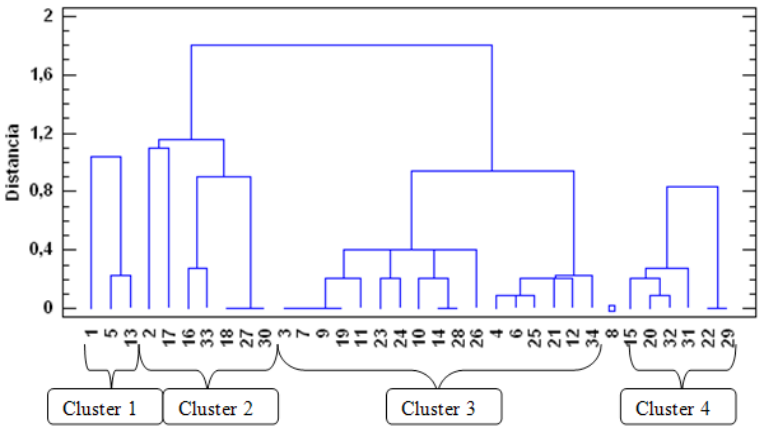

Fuente: Elaboración propia

\begin{tabular}{|c|c|c|}
\hline & $\begin{array}{l}\text { Grupo de } \\
\text { estudiantes }\end{array}$ & Caracteristicas \\
\hline \multirow{5}{*}{ 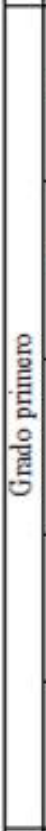 } & Desinformados & $\begin{array}{l}\text { Se agrupan } 6 \text { estudiantes, quienes se } \\
\text { caracterizan porque son los } \\
\text { estudiantes realizaronuna estimación } \\
\text { sin tener en cuenta la información } \\
\text { dada en el material, produciendo una } \\
\text { respuesta incoherente a la situación. }\end{array}$ \\
\hline & Des organizados & $\begin{array}{l}\text { En este se agrupan } 12 \text { estudiantes, } \\
\text { quienes se caracterizan por realizar } \\
\text { una estimación sin tener en cuenta la } \\
\text { información entregada pero agregan } \\
\text { datos poco relevantes en la respuesta } \\
\text { gráfica }\end{array}$ \\
\hline & Informados & $\begin{array}{l}\text { En este se agrupan } 3 \text { estudiantes, } \\
\text { quienes se caracterizan por que los } \\
\text { estudiantes realizan una estimación } \\
\text { de acuerdo al contexto y a la } \\
\text { información entregada, agregando } \\
\text { datos. }\end{array}$ \\
\hline & Bayesianos & $\begin{array}{l}\text { En este se agrupan } 12 \text { estudiantes } \\
\text { caracterizados por usan el } \\
\text { razonamiento bayesiano, donde a } \\
\text { partir de la información dada realizan } \\
\text { una estimación acorde a la situación. }\end{array}$ \\
\hline & Casos atipicos & $\begin{array}{l}\text { En este grupo se encuentran } 2 \\
\text { estudiantes que se caracterizan } \\
\text { porque presentan escases de } \\
\text { razonamiento bayesiano ya que no } \\
\text { estiman dada la informacion. }\end{array}$ \\
\hline \multirow{5}{*}{ 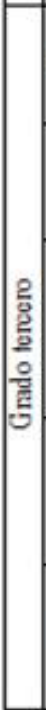 } & Desinformados & $\begin{array}{l}\text { En este, se agrupan } 6 \text { estudiantes, } \\
\text { caracterizindoseporque realizan una } \\
\text { estimación sin tener en cuenta la } \\
\text { información entregada. }\end{array}$ \\
\hline & Informados & $\begin{array}{l}\text { Se agrupan } 3 \text { estudiantes, quienes se } \\
\text { caracterizan porque realizaron una } \\
\text { estimación utilizando información } \\
\text { adicional. }\end{array}$ \\
\hline & $\begin{array}{c}\text { Escritores } \\
\text { Bayesianos }\end{array}$ & $\begin{array}{l}\text { Se agrupa } 17 \text { estudiantes } \\
\text { caracterizados por el uso del } \\
\text { razonamiento bayesiano enlo escrito, } \\
\text { donde a partir de la información } \\
\text { dada, realizanuna estimación acorde } \\
\text { a la situación. }\end{array}$ \\
\hline & Bayesianos & $\begin{array}{l}\text { Se agrupan } 7 \text { estudiantes, } \\
\text { caracterizadosporusar razonamiento } \\
\text { bayesiano, donde a partir de la } \\
\text { información dada, realizan una } \\
\text { estimación acorde a la situación. }\end{array}$ \\
\hline & Casos atipicos & $\begin{array}{l}\text { En este grupo se encuentra } 1 \\
\text { estudiante que se caracteriza por } \\
\text { presentarrazonamiento bayesiano ya } \\
\text { que estima de acuerdo a la } \\
\text { información dada. }\end{array}$ \\
\hline
\end{tabular}

Fuente: Elaboración propia 
grupos de 35 estudiantes cada uno, correspondientes a un diferente Cluster.

\section{Conclusiones}

El razonamiento bayesiano es un concepto que se debe construir mediante un proceso, donde el estudiante debe dejar de lado el pensamiento determinista, para qué empiece a ser consciente de la información proporcionada teniendo en cuenta sus condicionantes, entendiendo la importancia de la estimación en una situación aleatoria. Es allí donde la cultura estadística cobra gran importancia, ya que permite reflexionar estadísticamente frente a la cotidianidad

Se muestra que utilizar otro recurso diferente al algoritmo y utilizar el relato para la observación del razonamiento bayesiano, es una gran herramienta que permite ver más acciones, procedimientos y estimaciones que el algoritmo deja de lado. Este trabajo aporta a observar la importancia de que este proceso se realice desde los primeros años en la educación, mediante un recurso de fácil entendimiento a la población estudiada.

\section{Referencias}

Batanero, C., Ortiz, J. J., \& Serrano, L. (3 de Mayo de 2002). Investigación en didáctica de la probabilidad. Obtenido de UGR: http://www.ugr. es/ batanero/ARTICULOS/uNOiNVESTIGACION.pdf

Batanero, C. (2002). Probabilidad, grado de creencia y proceso de aprendizaje. XIII Jornadas Nacionales de Enseñanza y Aprendizaje de las Matemáticas. Granada: Federación Española de Profesores de Enseñanza de las Matemáticas.

Brousseau, G. (1986). Teoría de las situaciones didácticas. Madrid: s.n.

Diaz, C., \& Batanero, C. (3 de Mayo de 2006). ¿Cómo puede el Método Bayesiano Contribuir a la Investigación en Psicología y Educación? Obtenido de UGR: http://www.ugr.es/ batanero/ARTICULOS/Paradigma.pdf

García, J. M. (2011). Evaluación de conocimientos y recursos didácticos en la formación de profesores sobre probabilidad condicional. Granada.

Godino, B. C. (2006). Cómo puede el Método Bayesiano Contribuir a la Investigación en Psicología y. Recuperado el 26 de marzo de 2012, de http://www.ugr.es/ batanero/ ARTICULOS/Paradigma.pdf

Krauss, S., Bruckmaier, G., \& Martignon, L. (2010). Teaching young grownups how to use bayesian networks. ICOTS8, 6. 